Wild, EJ; Carroll, JB; (2012) HDBuzz: empowering patients through accessible education. Trends in Molecular Medicine , 18 (1) 1 - 3.

\title{
ARTICLE
}

\section{HDBuzz: empowering patients through accessible education}

\author{
Ed Wild ${ }^{1}$ and Jeffrey Carroll ${ }^{2}$
}

${ }^{1}$ UCL Institute of Neurology, National Hospital for Neurology and Neurosurgery, London, UK, WC1N 3BG.

${ }^{2}$ Harvard Medical School, Boston, USA, 02114.

Corresponding Author: Carroll, J. (jcarroll@chgr.mgh.harvard.edu)

\section{First paragraph ("abstract") 50-75 Words:}

Research and patient communities are firmly interdependent. Engaged patient communities provide biological samples and data that drive discoveries that, in turn, fuel the development of novel therapies. Historically, Huntington's disease (HD) has benefited from trusting interactions between scientists and patients. However, even for $\mathrm{HD}$, communication between the research and patient communities is suboptimal. The web platform HDBuzz was created to rectify this situation by providing accurate, accessible information on the latest HD research to patients and their supporters.

\section{The history}

The close relationship between patients and scientists developed from the begining of $H D$ research and has greatly furthered studies into the mechanisms of the disease. The genetic locus for HD was mapped to chromosome 4 in 1983 in two families, one American and one Venezuelan [1], whose pedigrees were collected by two large-scale patient observation efforts - the National Research Roster for Huntington Disease Patients and Families at Indiana University and the Venezuela Collaborative HD Project. Continued investigation led to the cloning of the causative gene in 1993 [2]. Since 1993 a number of large-scale observational projects have studied both symptomatic [3] and pre-symptomatic [4,5] mutation carriers. Predictive testing has become widespread [6], and reproductive options for HD families now include pre-natal screening that can prevent transmission of the mutant allele [7].

The cloning of the HD gene led to great excitement in some quarters that diseasemodifying therapies would be forthcoming. This excitement was tempered for others by the realization that the mutation occurred in a large $(340 \mathrm{kDa})$ and highly-conserved, yet novel, protein [2]. Clearly, future therapies would need to rest on a broad foundation of understanding both the function of the protein itself as well as the mechanism by which the mutation causes pathology.

In the intervening 18 years, knowledge of the precise genetic cause of HD has made the disease a fertile area for study. In addition to clinical data, studies in a vast array of models of the disease, from yeast [8] to primates [9], generate hundreds of publications per year. Many patients, encouraged by the speed of the remarkable series of experiments that led to the identification of the gene, have found the pace of subsequent progress from gene to effective treatments disappointing.

\section{The obstacles}


The HD community is extremely hungry for information about ongoing research, but any 'lay person' motivated enough to penetrate the paywalls of journal websites or attend exclusive scientific conferences is confronted by even more daunting barriers of jargon and the assumption of years of prior formal study. Furthermore, the vast array of scientific results presents a problem to patients: as non-experts, how can they judge which advances are relevant to them?

Another, more insidious, factor has contributed to patient disillusionment. Aware of the importance of piquing public interest in sustaining funding, university-based research organizations have embraced the practice of public relations. Scientific research publications on topics related to human disease are frequently accompanied, or preceded, by a press release touting the potential cures promised by this new breakthrough. These releases - often not written by non-scientists or 'enhanced' by press offices - have been said to "promote research that has uncertain relevance to human health" and fail to "provide key facts or acknowledge important limitations" [10]. These press releases, having passed into the public domain, may then be magnified further by sensationalist media reporting. Famously, one British newspaper has been accused of seeking to dichotomize all known substances into those that can either cause or cure cancer (http://www.guardian.co.uk/commentisfree/2010/oct/16/ben-goldacrebad-science-daily-mail-cancer). Speculative discussion in online communities such as blogs and discussion fora can have a multiplicative effect on the drawing of conclusions unwarranted by the primary research findings.

There have been positive research developments for HD patients, but successfully testing these therapies will require a large pool of potential volunteers, particularly as the distribution of sites for any given trial is not homogenous and inclusion/exclusion criteria are strict. There are now more trials for HD than ever before (http://clinicaltrials.gov/ct2/results?term=huntington\%27s), providing hope that meaningful therapies may be available soon. Recruitment for recent trials in HD has been disappointingly slow, but educating patients about HD drug trials appears to increase recruitment rates, supporting the hypothesis that a lack of reliable information is a major hurdle to successful trial recruitment [11].

\section{HDBuzz: bridging the communication gap}

Inspired by discussions with young people affected by HD at a youth group meeting of the European Huntington Disease Network, we recently developed and launched 'HDBuzz' (http://hdbuzz.net/) in an effort to help bridge this communication gap between the research and HD patient communities. HDBuzz is an online information source for people affected by HD and the community of carers that surrounds them. HDBuzz publishes short articles written in plain language that summarize recent clinical or basic research advances. Each article is written by a clinician or scientist expert in the field, but one with no connection to the work being discussed. Articles are signed by both the writer and editor, ensuring accountability for accuracy and legibility. Particular attention is given to placing each article within the context of the broader search for effective treatments, fostering a brand of realistic, pragmatic enthusiasm we refer to as 'substantive hope'.

All HDBuzz content is freely available under a Creative Commons license. In addition to the web platform, content can be syndicated via RSS and javascript feeds, email, Twitter (@HDBuzzfeed) and Facebook (HDBuzzfeed). Because most HD families do not live in 
English speaking countries [12], HDBuzz is translated into other languages. Thanks to a network of volunteer translators and a novel online interface, HDBuzz content is now available in Spanish, Portuguese, Italian, German, French, Polish, Danish and Dutch.

To avoid potential conflicts of interest, HDBuzz does not accept funding from any organization with a stake in a specific therapy. An international consortium of HD lay organizations provides funding to HDBuzz, with the understanding that it is completely independent editorially. The founding partners of HDBuzz were the HD Association (England \& Wales), Huntington Society of Canada and Huntington's Disease Society of America and since its launch, eight additional lay organizations have contributed towards its upkeep. This funding model provides maximum editorial independence for HDBuzz and removes even the appearance of bias towards any particular therapy.

Many lay organizations, including the HD Association (England \& Wales), Huntington Society of Canada and Huntington's Disease Society of America, use syndicated HDBuzz content to keep their communities informed about recent research news, freeing them to focus on the direct care they do best.

\section{Defining success}

HDBuzz is motivated by the idea that accurately communicating research to the affected community is a moral obligation of the scientific community: a work of science is not complete until this has been done. Meeting this obligation is the primary goal of HDBuzz and will be judged by the increased understanding of the scientific process in affected communities.

There are practical benefits of informed engagement for the entire HD community. Based on preliminary studies [11], it is hoped that careful education of HD families will lead to their more active participation in observational and clinical trials. Parsing the specific effects of HDBuzz in this effort will be difficult, but the goal of this practical motivation is to reduce recruitment time for new clinical trials and to rapidly fill ongoing observational trials with engaged and motivated participants. It is only by completing trials that we can close the circle of research and bring the fruits of basic and clinical science to patients.

\section{Results}

Since launching in January 2011 , traffic to the site has rapidly increased to over 56,000 visits a month, and more than 800 people have signed up to receive HDBuzz stories by email subscription (Figure 1; real-time statistics are available online at http://hdbuzz.net/statistics). Given the relative rarity of HD [13], this represents penetration into a significant proportion of the HD patient community in a very short time.

Through the web platform, users are encouraged to provide immediate feedback by clicking buttons labeled "Enjoyed" or "Didn't understand". Qualitative feedback suggests the message of cautious optimism is being communicated, even when covering complex material. In response to a recent story on genome editing, a reader commented, "great news and although a huge challenge its not impossible". Simultaneously grasping the excitement of recent developments and the difficulties in implementing them is precisely our goal. An article dealing with media reports reporting possible effects of cannabis 
and caffeine in HD prompted the comment "It clarifies many questions and also provide how we should interpret health news like this one".

\section{Beyond HDBuzz}

The early success of HDBuzz suggests that there is a significant unmet hunger for accurate research news in the patient community, and it seems unlikely that this appetite is limited to families afflicted by Huntington's Disease. Similar platforms, staffed by scientists with expertise in other clinical domains, may provide significant benefits to these areas. Our experience, and the web-based tools we have developed, may be useful for these efforts. The hope is that HDBuzz can serve as a template for how to bridge the scientific communication gap with obvious benefits to researchers and patients alike.

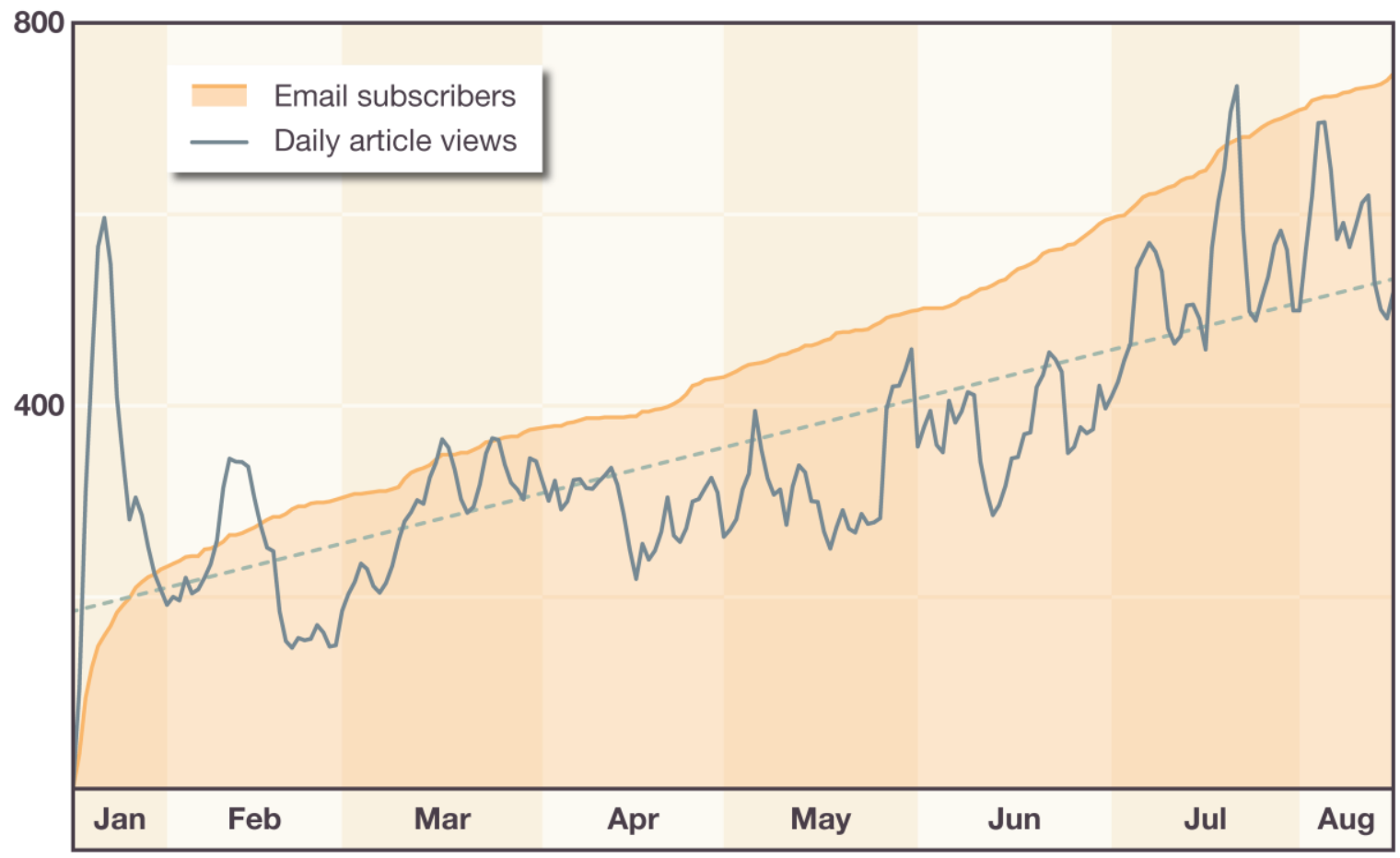

Figure Caption: Summary of email subscribers (orange) and daily article views (blue line, 5-day moving mean; dashed line, linear trend) at HDBuzz.net since launch on January 19, 2011. 


\section{References}

1 Gusella, J. F., Wexler, N. S., Conneally, P. M., Naylor, S. L., Anderson, M. A., Tanzi, R. E., Watkins, P. C., Ottina, K., Wallace, M. R., and Sakaguchi, A. Y. (1983). A polymorphic DNA marker genetically linked to Huntington's disease. Nature 306, 234-238.

2 The Huntington Disease Collaborative Research Group (1993). A novel gene containing a trinucleotide repeat that is expanded and unstable on Huntington"s disease chromosomes. The Huntington"s Disease Collaborative Research Group. Cell 72, 971-983.

3 The Huntington Study Group (1996). Unified Huntington's Disease Rating Scale: reliability and consistency. Huntington Study Group. Mov Disord 11, 136-142.

4 Paulsen, J. S., Hayden, M., Stout, J. C., Langbehn, D. R., Aylward, E., Ross, C. A., Guttman, M., Nance, M., Kieburtz, K., Oakes, D., et al. (2006). Preparing for preventive clinical trials: the Predict-HD study. Arch Neurol 63, 883-890.

5 Tabrizi, S. J., Scahill, R. I., Dürr, A., Roos, R. A., Leavitt, B. R., Jones, R., Landwehrmeyer, G. B., Fox, N. C., Johnson, H., Hicks, S. L., et al. (2011). Biological and clinical changes in premanifest and early stage Huntington's disease in the TRACK-HD study: the 12-month longitudinal analysis. Lancet Neurol 10, 31-42.

6 Benjamin, C. M., Adam, S., Wiggins, S., Theilmann, J. L., Copley, T. T., Bloch, M., Squitieri, F., McKellin, W., Cox, S., and Brown, S. A. (1994). Proceed with care: direct predictive testing for Huntington disease. Am J Hum Genet 55, 606-617.

7 Sermon, K., Seneca, S., De Rycke, M., Goossens, V., Van de Velde, H., De Vos, A., Platteau, P., Lissens, W., Van Steirteghem, A., and Liebaers, I. (2001). PGD in the lab for triplet repeat diseases - myotonic dystrophy, Huntington's disease and Fragile-X syndrome. Mol Cell Endocrinol 183 Suppl 1, S77-85.

8 Outeiro, T. F., and Muchowski, P. J. (2004). Molecular genetics approaches in yeast to study amyloid diseases. J. Mol. Neurosci. 23, 49-60.

9 Yang, S.-H., Cheng, P.-H., Banta, H., Piotrowska-Nitsche, K., Yang, J.-J., Cheng, E. C. H., Snyder, B., Larkin, K., Liu, J., Orkin, J., et al. (2008). Towards a transgenic model of Huntington's disease in a non-human primate. Nature 453, 921-924.

10 Woloshin, S., Schwartz, L. M., Casella, S. L., Kennedy, A. T., and Larson, R. J. (2009). Press releases by academic medical centers: not so academic? Ann. Intern. Med. 150, 613-618.

11 Goodman, L., Sia, C., Carnes, R., Vetter, L., Taubman, F., Venuto, C. S., McGarry, A., Kieburtz, K., and Agarwal, P. (2011). Advocacy Recruiting for Huntington's Disease Clinical Trials. PLoS Currents 3.

12 Kremer, B., Goldberg, P., Andrew, S. E., Theilmann, J., Telenius, H., Zeisler, J., Squitieri, F., Lin, B., Bassett, A., Almqvist, E., et al. (1994). A worldwide study of the Huntington's disease mutation. The sensitivity and specificity of measuring CAG 
repeats. N Engl J Med 330, 1401-1406.

13 Walker, F. O. (2007). Huntington's disease. Lancet 369, 218-228. 